Finney, D. J. (1951). J. gen. Microbiol. 5, 223-230.

\title{
The Statistical Analysis of Slope-Ratio Assays
}

\author{
By D. J. FINNEY \\ Lectureship in the Design and Analysis of Scientific Experiment, University of Oxford
}

SUMMARY: In the past, the statistical analysis of slope-ratio assays has failed to make specific tests of the validity of assumptions essential to the validity of the conclusions to be drawn, and has instead relied upon a composite test of residual variation. The technique introduced in this paper enables the analysis of variance of assay data to be developed in a manner analogous to that customary for parallel line assays. The paper illustrates the calculations for a 5-point assay, and shows how the analysis of variance, completed with the aid of a set of orthogonal coefficients, gives the required validity tests. Similar analyses for other assay designs, symmetrical and unsymmetrical, are briefly described, and tables of the orthogonal coefficients for 5-, 7-, 9-, and 11-point designs are given. A final section discusses the need for validity tests in biological assays.

In many microbiological assays, the response measured for the test organism shows a linear regression on the dose. The relative potency of two preparations is then estimated by the ratio of the two regression coefficients. The statistical analysis of such a slope-ratio assay was first described in 1945, and improved versions have since been published (Wood, 1946; Finney, 1947). Wood \& Finney (1947) discussed the design of slope-ratio assays, and recommended the general adoption of symmetrical $(2 k+1)$-point designs: in these, tests are made on 'blanks' (i.e. zero dose) and on $k$ equally spaced doses of the standard and test preparations, using the same replication for all doses. The 5-point design is particularly convenient and useful.

For parallel line assays, in which the response shows a linear regression on the logarithm of dose, tests of the validity of basic assumptions on parallelism and linearity can be made very simply by the use of orthogonal coefficients (Emmens, 1948; Burn, Finney \& Goodwin, 1950). The purpose of the present paper is to describe a similar procedure for slope-ratio assays, by means of which the analysis of variance is made much more informative than in the past. In particular, the analysis distinguishes clearly between tests for fundamental and for statistical validity: without these tests the drawing of conclusions from a biological assay is fraught with danger, and without distinction between them the investigator does not know whether an apparently invalid assay needs a modified analysis or is based on unsound premisses.

\section{The 5-point assay}

Table 1 records the responses for an assay of riboflavin in a sample of malt (Wood, 1946), expressed in units of $0.05 \mathrm{ml} .0 \cdot 1 \mathrm{~N}-\mathrm{NaOH}$ so as to give convenient whole numbers. When the mean responses are plotted against dose (Fig. 1), they are seen to lie close to two straight lines intersecting at zero dose. The statistical analysis will consist in estimating the slopes of the best-fitting lines, and testing their adequacy as a representation of the data. 
As Wood showed in an appendix to his paper, the regression coefficients may be obtained by formal estimation of a multiple linear regression equation,

$$
Y=a+b_{S} x_{S}+b_{T} x_{T}
$$

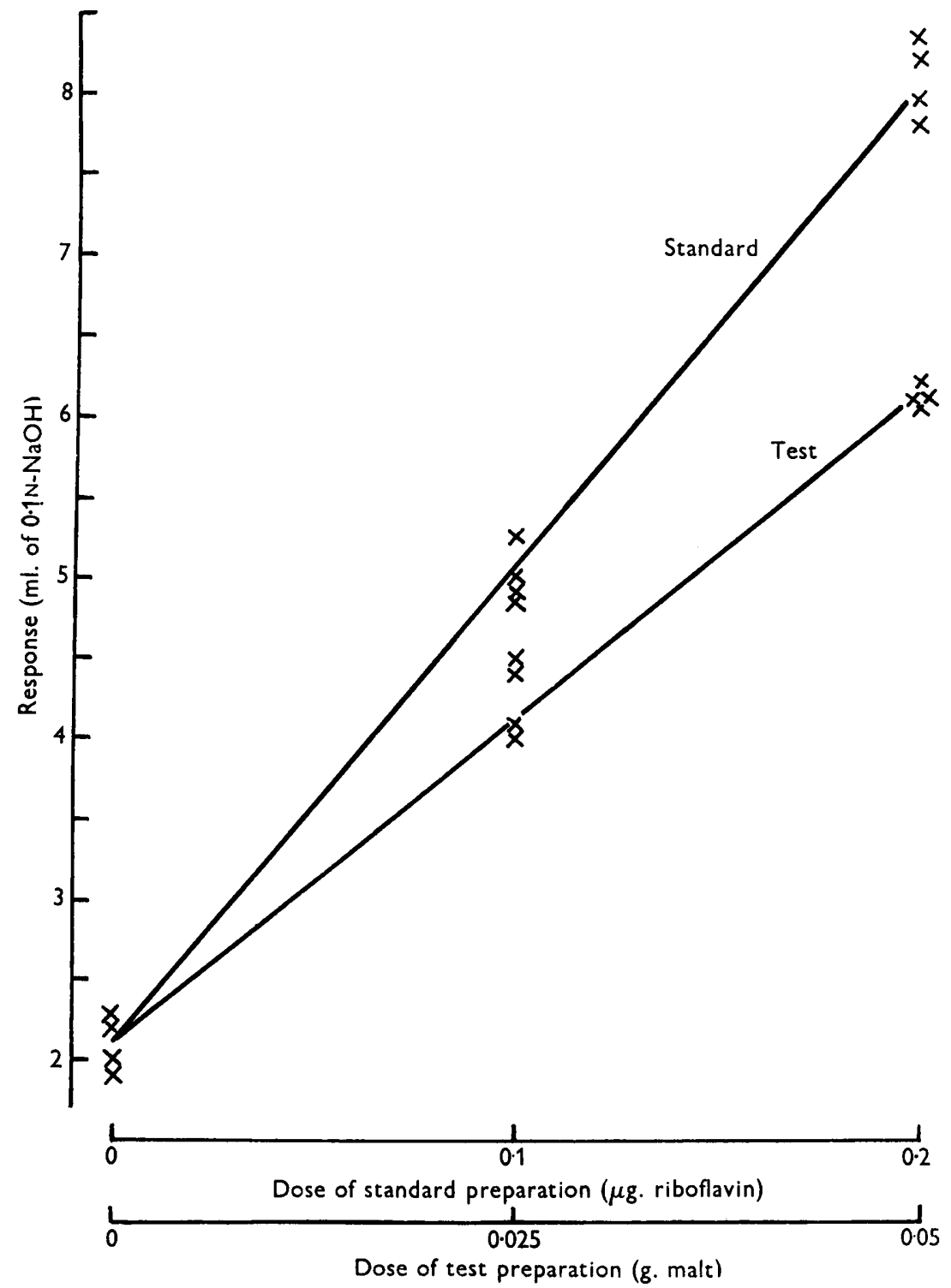

Fig. 1. Response diagram for an assay of riboflavin in malt.

Here $x_{S}$ represents the dose of the standard preparation, $x_{T}$ the dose of the test preparation, and $Y$ is the average response to $x_{S}$ and $x_{T}$ simultaneously; in ordinary assay practice, the two preparations are always given separately, so that for every response one or other of $x_{S}, x_{T}$ is zero. The values of $x_{S}, x_{T}$ in the riboflavin assay are shown at the heads of the columns in Table 1. For 
Table 1. Responses in an assay of riboflavin in malt

One unit of standard preparation $=0 \cdot 2 \mu \mathrm{g}$. riboflavin. One unit of test preparation $=0.05 \mathrm{~g}$. malt. Responses are measured in units of $0.05 \mathrm{ml} .0 \cdot 1 \mathrm{~N}-\mathrm{NaOH}$.

\begin{tabular}{ccccc} 
Blanks & \multicolumn{2}{c}{ Standard } & $x_{S}$ & Test \\
$x_{S}=0$ & $x_{S}=\frac{1}{2}$ & $x_{s}=1$ & $x_{S}=0$ & $x_{S}=0$ \\
$x_{r}=0$ & $x_{T}=0$ & $x_{T}=0$ & $x_{T}=\frac{1}{2}$ & $x_{T}=1$ \\
38 & 97 & 167 & 80 & 121 \\
45 & 100 & 164 & 88 & 124 \\
40 & 105 & 159 & 90 & 122 \\
44 & 98 & 156 & 82 & 122 \\
167 & 400 & 646 & 340 & 489
\end{tabular}

the estimation of the regression coefficients, $b_{S}$ and $b_{T}$ (which represent the increase in response per unit increase in dose of the standard and test preparations), sums of squares and products of the deviations of $x_{S}, x_{T}, y$ from their respective mean values are needed. Though $S(y-\bar{y})^{2}$ is not required immediately, it may conveniently be computed at the same time as the others. The calculations of these sums, which are of a type familiar to all who have calculated standard errors and correlation coefficients, proceed as

$$
S\left(x_{S}-\bar{x}_{S}\right)(y-\bar{y})=S x_{S} y-\left(S x_{S}\right)(S y) / 5 n,
$$

where $S($ ) represents summation over all responses and $n$ is the number of responses measured for each dose (here $n=4)$. They may be summarized:

\begin{tabular}{cccrr}
$\left(x_{S}^{2}\right),\left(x_{T}^{2}\right)$ & $\left(x_{s} x_{Y}\right)$ & $\left(x_{s} y\right)$ & $\left(x_{T} y\right)$ & \multicolumn{1}{c}{$\left(y^{2}\right)$} \\
$5 \cdot 0$ & $0 \cdot 0$ & $846 \cdot 0$ & $659 \cdot 0$ & $\mathbf{2 4 0 , 1 9 8 \cdot 0}$ \\
$1 \cdot 8$ & $1 \cdot 8$ & $612 \cdot 6$ & $612 \cdot 6$ & $\mathbf{2 0 8 , 4 8 8 \cdot 2}$ \\
$3 \cdot 2$ & $-1 \cdot 8$ & $\mathbf{2 3 3 \cdot 4}$ & $\mathbf{4 6 \cdot 4}$ & $\mathbf{3 1 , 7 0 9 \cdot 8}$
\end{tabular}

The first two lines correspond to the two terms on the right-hand side of the last equation, the third is their difference. The regression coefficients are the solutions of the equations

$$
3 \cdot 2 b_{S}-1 \cdot 8 b_{T}=233 \cdot 4, \quad-1 \cdot 8 b_{S}+3 \cdot 2 b_{T}=46 \cdot 4,
$$

and are therefore $\quad b_{S}=118 \cdot 629, b_{T}=81 \cdot 229$.

Alternatively, $b_{S}$ and $b_{T}$ can be calculated directly from the five dose totals at the foot of Table 1: if the dose totals be denoted by $C$ (for the blanks), $S_{1}, S_{2}$, $T_{1}, T_{2}$, then

$$
b_{S}=\frac{2}{35 n}\left(-15 C+S_{1}+17 S_{2}-6 T_{1}+3 T_{2}\right),
$$

and

$$
b_{T}=\frac{2}{35 n}\left(-15 C-6 S_{1}+3 S_{2}+T_{1}+17 T_{2}\right)
$$

which give the same numerical values as before. The advantage in this form of calculation is slight, however, because $S\left(x_{S}-\bar{x}_{S}\right)(y-\bar{y})$ and $S\left(x_{T}-\bar{x}_{T}\right)(y-\bar{y})$ are required in the construction of the analysis of variance.

The analysis of variance (Table 2) must next be completed. The total sum of squares of deviations for the responses is easily divided into components between doses (4 degrees of freedom) and within doses $\overline{(n-1}$ degrees of freedom 
from each dose); the sum of squares between doses is calculated from the five dose totals, in the usual manner. The quantity

$$
\begin{aligned}
b_{S} S\left(x_{S}-\bar{x}_{S}\right)(y-\bar{y})+b_{T} S\left(x_{T}-\bar{x}_{T}\right)(y-\bar{y}) & =118.629 \times 233 \cdot 4+81 \cdot 229 \times 46 \cdot 4 \\
& =31,456 \cdot 9
\end{aligned}
$$

is the part of the sum of squares between doses accounted for by the regression. The remainder may be split into two components, with 1 degree of freedom each, the first dependent upon the deviation of the mean response for the

Table 2. Analysis of variance for the riboflavin assay

\begin{tabular}{lrrr} 
Adjustment for mean & \multicolumn{3}{c}{$\mathbf{2 0 8 , 4 8 8 \cdot 2}$} \\
Nature of variation & D.F. & Sum of squares & Mean square \\
Regression & 2 & $\mathbf{3 1 , 4 5 6 \cdot 9}$ & - \\
Blanks & 1 & $2 \cdot 2$ & $\mathbf{2 \cdot 2}$ \\
Intersection & 1 & $34 \cdot 2$ & $\mathbf{3 4 \cdot 2}$ \\
Between doses & 4 & $\mathbf{3 1 , 4 9 3 \cdot 3}$ & - \\
Error & 15 & $\mathbf{2 1 6 \cdot 5}$ & $\mathbf{1 4 \cdot 4 3}$ \\
Total & 19 & $31,709 \cdot 8$ & -
\end{tabular}

blanks from regressions fitted to the non-zero doses and the second dependent upon the deviation of the point of intersection of regressions fitted separately to the two preparations from the zero level of dose. The first, to be described as Blanks, is calculated as $L_{C}^{\prime 2} / 14 n$, where

$$
L_{C}=2 C-2 S_{1}+S_{2}-2 T_{1}+T_{2},
$$

provides a test of statistical validity, in particular of whether the assumption of linear regression down to $x_{S}=x_{T}=0$ is contradicted by the data. The second, to be described as Intersection, is calculated as $L_{C}^{\prime 2} / 10 n$, where

$$
L_{C}^{\prime}=2 S_{1}-S_{2}-2 T_{1}+T_{2}
$$

provides a test of fundamental validity of the assay, for unless regressions fitted separately to the standard and test data intersect at $x_{S}=x_{T}=0$ (except for deviations attributable to random variation) the whole basis of the assay is faulty (cf. Jerne \& Wood, 1949). For the data of Table 1,

$$
L_{C}=-11 \text {, with divisor } 56 \text {, }
$$

and

$$
L_{C}^{\prime}=-37 \text {, with divisor } 40 .
$$

The three components, regression, blanks, and intersection, add exactly to the sum of squares between doses.

In this instance, neither the blanks nor the intersection component is significantly greater than the error mean square, and estimation of the relative potency may proceed. One unit of the test preparation is estimated to contain $\boldsymbol{R}=b_{T} / b_{S}=\mathbf{0 . 6 8 5}$ units of the standard. Fiducial limits may be found by formulae described elsewhere (Wood, 1946; Finney, 1947), and are 0.646, 0.724 for the $95 \%$ probability level. Reference to the definition of units at the head of Table 1 then shows that $1 \mathrm{~g}$. malt is estimated to contain $2 \cdot 74 \mu \mathrm{g}$. riboflavin, with $95 \%$ fiducial limits at $2 \cdot 58$ and $2 \cdot 90 \mu \mathrm{g}$. 
Wood (1946) proposed to use the deviations of the quantities

$$
L_{S}=C-2 S_{1}+S_{2} \text { and } L_{T}=C-2 T_{1}+T_{2}
$$

from zero as tests of validity, these representing tests of the linearity of regressions for each preparation separately, using the blanks in turn with each. The method proposed here, though based upon the general procedure described below, is equivalent to testing $\left(L_{S}+L_{T}\right)$ and $\left(L_{S}-L_{T}\right)$. The advantages for the present proposal are that it separates the tests into one of statistical and one of fundamental validity, that it fits into the structure of the analysis of variance, and that it generalizes to the $(2 k+1)$-point design. In practice, the decisions taken in respect of any particular set of data will usually be the same if based on Wood's analysis instead of on that advocated here.

\section{Other assay designs}

The scheme for the analysis of the sum of squares between doses which has been illustrated for the symmetrical 5-point assay may be extended to other designs. For the 9-point design, for example, having $n$ tests at four doses of each preparation as well as for the blanks, the sum of squares for blanks is $L_{C}^{2} / 28 n$, where

$$
L_{C}=4 C-2\left(S_{1}+T_{1}\right)-\left(S_{2}+T_{2}\right)+\left(S_{4}+T_{4}\right)
$$

and that for intersection is $L_{C}^{\prime 2} / 12 n$, where

$$
L_{C}^{\prime}=2\left(S_{1}-T_{1}\right)+\left(S_{2}-T_{2}\right)-\left(S_{4}-T_{4}\right) .
$$

These, together with the sum of squares for regression found in the usual manner, account for 4 of the 8 degrees of freedom between doses. The remainder can be partitioned into quadratic and cubic components for each preparation separately, using contrasts

$$
S_{1}-S_{2}-S_{3}+S_{4} \text { and } T_{1}-T_{2}-T_{3}+T_{4}
$$

with divisor $4 n$, and

$$
-S_{1}+3 S_{2}-3 S_{3}+S_{4} \text { and }-T_{1}+3 T_{2}-3 T_{3}+T_{4}
$$

with divisor $20 \mathrm{n}$. The coefficients for these are the same as are used for quadratic and cubic terms in the more familiar parallel line assays, except that the two preparations are examined separately. For most purposes, a composite test of the residual curvature components comprised in these 4 degrees of freedom would suffice, but the full analysis may sometimes assist the understanding of a deviation from linearity.

The general formulae for a symmetrical $(2 k+1)$-point assay, with $n$ responses measured for each dose, have been developed and will be described elsewhere. For $k=1$, the calculations are trivial, as the regression coefficients are found from the differences between the responses to the preparations and that for the blanks; Wood \& Finney (1947) have advised against the use of this design because of its complete lack of validity tests. Tables 3-6 assist the calculations for designs with $k=2,3,4$, or 5 , and their use will be understood by comparing them with the instructions already given for the 5-point and 9-point designs. The regression coefficients are to be found exactly as described for the riboflavin 
assay (first method), by forming sums of squares and products and solving two linear equations. The expression

$$
b_{S} S\left(x_{S}-\bar{x}_{S}\right)(y-\bar{y})+b_{T} S\left(x_{T}-\bar{x}_{T}\right)(y-\bar{y})
$$

always gives the sum of squares (2 degrees of freedom) for the regression. From the dose totals are calculated the blanks and intersection components as well as components for quadratic, cubic, and quartic curvature (where these can be formed). Provided that the assay is not demonstrated to be invalid, formulae

Table 3. Orthogonal coefficients for a 5-point slope-ratio assay

\begin{tabular}{|c|c|c|c|c|c|c|}
\hline \multirow[b]{2}{*}{$\begin{array}{c}\text { Component } \\
\boldsymbol{L}_{\sigma} \\
\boldsymbol{L}_{\sigma}^{\prime}\end{array}$} & \multirow[b]{2}{*}{$\begin{array}{c}\text { Blanks } \\
\text { C } \\
2 \\
0\end{array}$} & \multicolumn{2}{|c|}{ Standard } & \multicolumn{2}{|c|}{ Test } & \multirow[b]{2}{*}{$\begin{array}{l}\text { Divisor } \\
14 n \\
10 n\end{array}$} \\
\hline & & $\begin{array}{r}S_{1} \\
-2 \\
2\end{array}$ & $\begin{array}{r}S_{2} \\
1 \\
-1\end{array}$ & $\begin{array}{r}T_{1} \\
-2 \\
-2\end{array}$ & $\begin{array}{l}T_{2} \\
\cdot \quad 1 \\
1\end{array}$ & \\
\hline
\end{tabular}

Table 4. Orthogonal coefficients for a 7-point slope-ratio assay

\begin{tabular}{|c|c|c|c|c|c|c|c|c|}
\hline \multirow[b]{2}{*}{ Component } & \multirow{2}{*}{$\begin{array}{c}\text { Blanks } \\
\quad C\end{array}$} & \multicolumn{3}{|c|}{ Standard } & \multicolumn{3}{|c|}{ Test } & \multirow[b]{2}{*}{ Divisor } \\
\hline & & $S_{1}$ & $S_{2}$ & $S_{3}$ & $T_{1}$ & $T_{2}$ & $T_{3}$ & \\
\hline $\boldsymbol{L}_{\sigma}$ & 6 & -4 & -1 & $\mathbf{2}$ & -4 & -1 & 2 & $78 n$ \\
\hline$L_{0}^{\prime}$ & $\mathbf{0}$ & 4 & 1 & -2 & -4 & -1 & 2 & $42 n$ \\
\hline Quadratic $(S)$ & $\mathbf{0}$ & 1 & -2 & 1 & 0 & $\mathbf{0}$ & $\mathbf{0}$ & $6 n$ \\
\hline Quadratic $(T)$ & 0 & 0 & o & $\mathbf{0}$ & 1 & -2 & 1 & $6 n$ \\
\hline
\end{tabular}

below the tables may be used to assess the precision of $R\left(=b_{T} / b_{S}\right)$, the estimate of relative potency. The first two equations give the variances of $b_{S}$ and $b_{T}$ and their covariance, and the third gives the variance of $R$. In these formulae, $s^{2}$ is the error mean square in the analysis of variance and $b_{S}, b_{T}$ are supposed to relate to scales of measurement for doses that make the highest dose of each preparation equal to 1 unit. The formula for $V(R)$ is applicable only if $b_{S}^{2} / V\left(b_{S}\right)$ is large (say greater than 100); if this condition is not satisfied, fiducial limits to $R$ must be found by formula (32) given by Finney (1947).

The same principle may be applied to the analysis of unsymmetrical assays, such as that first used as an illustration of the slope-ratio procedure (Finney, 1945, 1947). The square for blanks is then most easily found by introducing an auxiliary variate $x_{O}$ which takes the value 1 for all blank responses, $O$ for all others; $x_{O}$ is used formally as though it were a measure of dose for a third 'preparation'. The difference between the sum of squares with 3 degrees of freedom for a multiple regression on $x_{O}, x_{S}, x_{T}$, and that with 2 degrees of freedom for the regression on $x_{S}, x_{T}$ is the square for blanks. A sum of squares with 4 degrees of freedom may be formed by finding the sum of squares (2 degrees of freedom) between blanks, the total of all responses to the standard preparation, and the total of all responses to the test preparation, and adding to it squares for two linear regressions calculated from each preparation inde- 
pendently, ignoring the blanks; the difference between this and the sum of squares with 3 degrees of freedom for regression on $x_{O}, x_{S}, x_{T}$ is the square for intersection. The equivalence of this apparently arbitrary procedure to that used earlier may be demonstrated by elementary (though laborious) algebra.

Table 5. Orthogonal coefficients for a 9-point slope-ratio assay

\begin{tabular}{|c|c|c|c|c|c|c|c|c|c|c|}
\hline \multirow[b]{2}{*}{ Component } & \multirow{2}{*}{$\begin{array}{l}\text { Blanks } \\
\quad C\end{array}$} & \multicolumn{4}{|c|}{ Standard } & \multicolumn{4}{|c|}{ Test } & \multirow[b]{2}{*}{ Divisor } \\
\hline & & $S_{1}$ & $\boldsymbol{S}_{2}$ & $S_{3}$ & $S_{4}$ & $T_{1}$ & $\boldsymbol{T}_{2}$ & $T_{3}$ & $T_{4}$ & \\
\hline$L_{c}$ & 4 & -2 & -1 & $\mathbf{0}$ & $\mathbf{1}$ & -2 & -1 & $\mathbf{0}$ & 1 & $28 n$ \\
\hline$L_{c}^{\prime}$ & $\mathbf{0}$ & 2 & 1 & 0 & -1 & -2 & -1 & 0 & $\mathbf{1}$ & $12 n$ \\
\hline Quadratic (S) & 0 & 1 & -1 & -1 & 1 & $\mathbf{0}$ & $\mathbf{0}$ & $\mathbf{0}$ & 0 & $4 n$ \\
\hline Quadratic $(T)$ & 0 & 0 & o & o & 0 & 1 & -1 & -1 & 1 & $4 n$ \\
\hline Cubic $(S)$ & 0 & -1 & 3 & -3 & 1 & $\mathbf{0}$ & $\mathbf{0}$ & $\mathbf{0}$ & 0 & $20 n$ \\
\hline Cubic $(T)$ & 0 & $\mathbf{0}$ & 0 & o & 0 & -1 & $\mathbf{3}$ & -3 & $\mathbf{1}$ & $20 n$ \\
\hline
\end{tabular}

Table 6. Orthogonal coefficients for an 11-point slope-ratio assay

\begin{tabular}{|c|c|c|c|c|c|c|c|c|c|c|c|c|}
\hline & & \multicolumn{5}{|c|}{ Standard } & \multicolumn{5}{|c|}{ Test } & \multirow[b]{2}{*}{ Divisor } \\
\hline Component & $C$ & $S_{1}$ & $S_{2}$ & $S_{3}$ & $S_{4}$ & $S_{5}$ & $T_{1}$ & $T_{2}$ & $T_{3}$ & $T_{4}$ & $T_{5}$ & \\
\hline $\boldsymbol{L}_{c}$ & 20 & -8 & -5 & -2 & 1 & 4 & -8 & -5 & -2 & 1 & 4 & $620 n$ \\
\hline $\boldsymbol{L}_{c}^{\prime}$ & 0 & $\mathbf{8}$ & $\mathbf{5}$ & $\mathbf{2}$ & -1 & -4 & -8 & -5 & -2 & $\mathbf{1}$ & 4 & $220 n$ \\
\hline Quadratic (S) & $\mathbf{0}$ & 2 & -1 & -2 & -1 & 2 & o & 0 & $\mathbf{0}$ & $\mathbf{0}$ & $\mathbf{0}$ & $14 n$ \\
\hline Quadratic (T) & $\mathbf{0}$ & 0 & o & 0 & o & o & 2 & -1 & -2 & -1 & 2 & $14 n$ \\
\hline Cubic $(S)$ & $\mathbf{0}$ & -1 & 2 & 0 & -2 & 1 & o & 0 & $\mathbf{0}$ & $\mathbf{0}$ & $\mathbf{0}$ & $10 n$ \\
\hline Cubic $(T)$ & 0 & o & 0 & 0 & 0 & 0 & -1 & 2 & $\mathbf{0}$ & -2 & 1 & $10 n$ \\
\hline Quartic $(S)$ & $\mathbf{0}$ & 1 & -4 & 6 & -4 & 1 & 0 & 0 & 0 & o & 0 & $70 n$ \\
\hline Quartic (T) & $\mathbf{0}$ & $\mathbf{0}$ & o & $\mathbf{0}$ & $\mathbf{0}$ & $\mathbf{0}$ & 1 & -4 & 6 & -4 & 1 & $70 n$ \\
\hline
\end{tabular}

$$
V\left(b_{S}\right)=V\left(b_{T}\right)=\frac{380 s^{2}}{341 n} ; \operatorname{Cov}\left\{b_{S}, b_{T}\right\}=\frac{225 s^{2}}{341 n} ; V(R)=\frac{10 s^{2}\left(38-45 R+38 R^{2}\right)}{341 n b_{S}^{2}}
$$

\section{Validity tests}

The reader may wonder what is the use of this apparently complicated analysis, and may inquire whether it is really necessary. Certainly it adds nothing to the precision of the estimate of potency to be quoted as the conclusion from an assay. Any conclusion, however, rests upon certain assumptions about the nature of the preparations assayed and the character of the dose-response relationship (Jerne \& Wood, 1949). If these assumptions are untrue, the assay may be fundamentally invalid, or its statistical analysis may need radical alteration before any conclusion can legitimately be drawn. The experienced statistician may often be able to dispense with explicit tests of the validity of these assumptions, because he can see by inspection of the data that no significant evidence of invalidity is present; nevertheless, he must have at his command methods of testing for use in critical cases where he cannot trust his judgement. The user of assay techniques whose statistical experience is limited needs to be more cautious about the acceptance of basic assumptions without explicit and objective tests; for him especially, validity tests such as have been described may be important and frequently used. Indeed, validity 
tests should be regarded as an essential feature of biological assays, never to be neglected merely because they are laborious; the only excuse that can rightly be made for omission of explicit tests is that the results of these could be foretold with certainty, without performing the arithmetical operations. Though the statistical analysis of slope-ratio assays is more laborious than that of parallel line assays, adoption of a symmetrical design eliminates much of the labour and is theoretically desirable; moreover, the labour lies chiefly in the calculation of the regression coefficients, and, for the more important symmetrical designs, as shown above, the validity tests can be based on standard sets of orthogonal coefficients just as easily as for parallel line assays.

\section{REFERENCES}

Burn, J. H., Finney, D. J. \& Goodwin, L. G. (1950). Biological Standardization. London: Oxford University Press.

Emmens, C. H. (1948). Principles of Biological Assay. London: Chapman and Hall.

FinNey, D. J. (1945). The microbiological assay of vitamins: the estimate and its precision. Quart. J. Pharm. 18, 77-82.

Finney, D. J. (1947). The principles of biological assay. J. R. statist. Soc. (Suppl.), 9, 46-91.

JERnE, N. K. \& Wood, E. C. (1949). The validity and meaning of the results of biological asays. Biometrics, 5, 273-99.

WooD, E. C. (1946). The theory of certain analytical procedures, with particular reference to micro-biological assays. Analyst, 71, 1-14.

Woon, E. C. \& FinNey, D. J. (1947). The design and statistical analysis of microbiological assays. Quart. J. Pharm. 19, 112-27.

(Received 2 May 1950) 